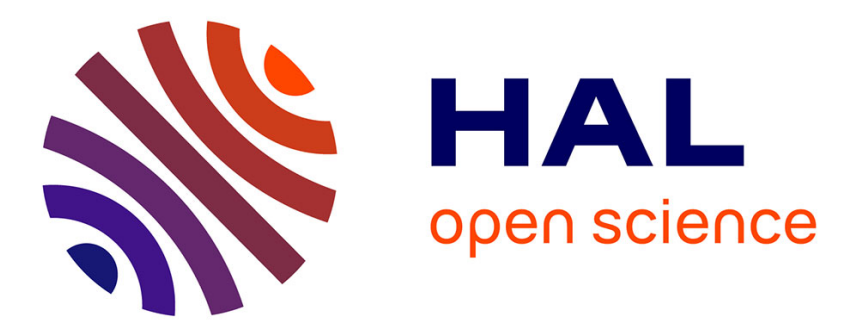

\title{
The influence of different loads on the remodeling process of a bone and bio-resorbable material mixture with voids
}

\author{
Ivan Giorgio, Ugo Andreaus, Angela Madeo
}

\section{- To cite this version:}

Ivan Giorgio, Ugo Andreaus, Angela Madeo. The influence of different loads on the remodeling process of a bone and bio-resorbable material mixture with voids. Continuum Mechanics and Thermodynamics, 2016, 28 (1-2), pp.21-40. 10.1007/s00161-014-0397-y · hal-01094868

\section{HAL Id: hal-01094868 \\ https://hal.science/hal-01094868}

Submitted on 14 Dec 2014

HAL is a multi-disciplinary open access archive for the deposit and dissemination of scientific research documents, whether they are published or not. The documents may come from teaching and research institutions in France or abroad, or from public or private research centers.
L'archive ouverte pluridisciplinaire HAL, est destinée au dépôt et à la diffusion de documents scientifiques de niveau recherche, publiés ou non, émanant des établissements d'enseignement et de recherche français ou étrangers, des laboratoires publics ou privés. 


\title{
The Influence of Different Loads on the Remodeling Process of a Bone and Bio-Resorbable Material Mixture with Voids
}

\author{
Ivan Giorgio · Ugo Andreaus · Angela Madeo
}

Received: date / Accepted: date

\begin{abstract}
A model of a mixture of bone tissue and bio-resorbable material with voids was used to numerically analyze the physiological balance between the processes of bone growth and resorption and artificial material resorption in a plate-like sample. The adopted model was derived from a theory for the behavior of porous solids in which the matrix material is linearly elastic and the interstices are void of material. The specimen - constituted by a region of bone living tissue and one of bio-resorbable material - was acted by different in-plane loading conditions, namely pure bending and shear. Ranges of load magnitudes were identified within which physiological states become possible. Furthermore, the consequences of applying different loading conditions are examined at the end of the remodeling process. In particular, maximum value of bone and material mass densities, and extensions of the zones where bone is reconstructed were identified and compared in the two different load conditions. From the practical view point, during surgery planning and later rehabilitation some choice of the following parameters is given: porosity of the graft, material characteristics of the graft, and adjustment of initial mixture tissue/bio-resorbable material and later, during healing and remodelling, optimal loading conditions.
\end{abstract}

Keywords Bio-mechanics · bone tissue · artificial bio-resorbable material · formation/resorption processes $\cdot$ continuum mixture model

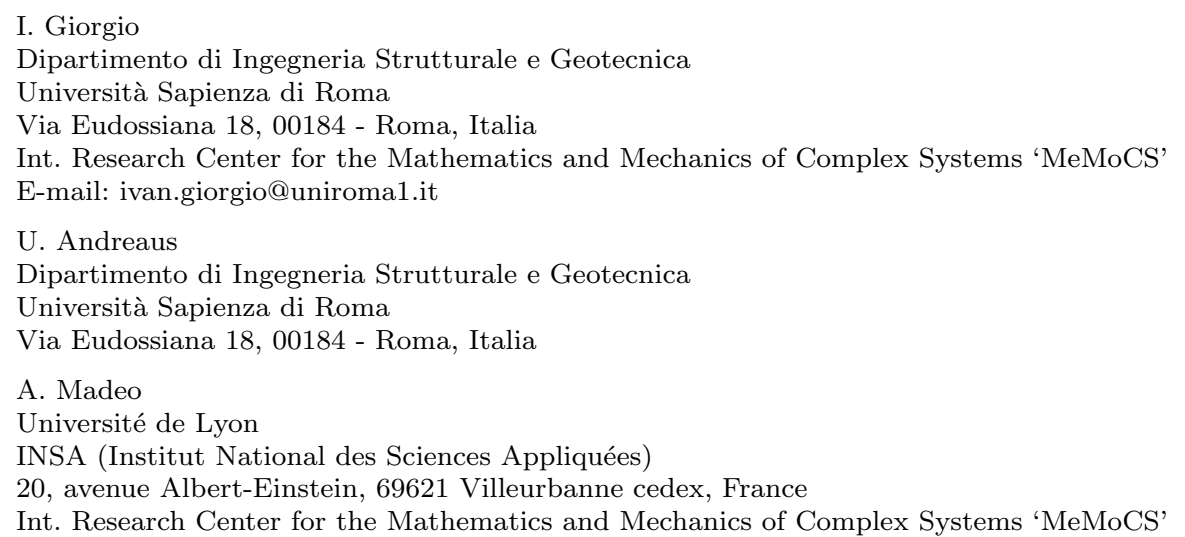




\section{Introduction}

Bone tissue is a dynamic system with life-lasting functional adaptation capabilities to environmental stresses. It is continuously remodeled by the coordinated action of bone-resorbing osteoclasts and boneforming osteoblasts. The balance between bone resorption and bone formation determines the distribution of mass density of bone. The activity of osteoclasts and osteoblasts is conjectured to be triggered by signals from sensor cells, which are sensitive also to mechanical causes, such as the density of strain energy [48]. However, in the case of extensive bone defects, such as bone fracture, pathological process, surgery infection, etc., this self-healing mechanism for the reconstruction of large bone segments is not feasible. Promoting the repair of large-sized defects can be obtained by introducing resorbable bio-materials. Then, bone formation and scaffold resorption are to be evaluated in order to trace the progressive osteoclastic resorption till the segmental defect is hopefully filled with newly formed bone. Recently, it is thought that the implant grafted onto bone must be designed with porous materials. These have a structure made up of a solid matrix and voids which let bone tissue grow inside the artificial material, as stated by $L i$ et al. [50]. Unfortunately, the grafting of implants is possible only when the grafted bone is not subject to intense loads, as discussed by Farrar [30]. The fatigue behaviour of bone cement was investigated by Soltész [73] with respect to the influence of loading conditions performing tests under simulated physiological conditions. Chun et al. [17] investigated the effect of different abutment types on the stress distribution in bone with inclined loads using Finite Element analysis and concluded that different load transfer mechanisms have significant influence on the stress distribution in bone. Andreaus et al. [6] numerically analyzed the stress shielding of the femoral bone with an artificial hip replacement and showed evidence that, when comparing different loading conditions, namely walking with stair climbing, for stresses and strain energy density, maxima and minima values change dramatically. Andreaus and Colloca [1] proposed a Finite Element approach for analyzing the structural behavior of hip joint prostheses under physiological loadings and boundary conditions, which allows the prediction of micromotion initiation. Two loading cases, namely walking and stair climbing, were applied to investigate the effect of different loading conditions on the shear strain patterns. Bevill et al. [10] tested cores of human vertebral trabecular bone under two different boundary conditions and loading modes (compression and torsion). High-resolution Finite Element models that explicitly modeled the different experimental conditions were constructed and sensitivity studies were performed to quantify errors arising from uncertainties between model and experiment. Shi et al. [72] used microstructural Finite Element models to compute regions of tissue level yielding in trabecular bone samples. The morphology, number, and mean volume of the yielded regions were quantified under two loading conditions, namely compression parallel and perpendicular to the principal material axis of the trabecular bone architecture. They concluded that localized high strains consistent with bending rather than axial deformation of struts occur at the tissue level. Fatihhi et al. [31] observed that the relationship between morphological parameter and different type of loading orientation on elastic behavior and yielding of trabecular may provide insight towards osteoporotic bone losses during normal activities. They attempted to predict the elastic and failure behavior of different loading modes (tensile and compression) on anatomic sites and morphological indices through Finite Element simulation. Results demonstrated differences between yield behaviors on anatomic sites were reflected onto the morphological indices and the type of loading modes.

In papers $[5,3,2,4]$, it is assumed that the bone remodelling rule is represented by the classical proportional-integral-derivative (PID) control, applied to the error signal between the actual Strain Energy Density (SED) and a SED target to be optimized; this target has to be determined from a normal equilibrium density distribution. The underlying premise is that the mass should be distributed in an efficient way, therefore using a minimum amount of material to accomplish the mechanical function, in which stiffness and mass are conflicting objectives. In a similar way, Olsson and Klarbring [63] impose a 'target angle' to be reached when the remodeling process is complete, in order to ensure that their dynamical equations have a unique solution. 
Some authors assume that a simple network of sensors measures the mechanical stimulus and 'directs' the actions of active cells. In general, the graft material is absolutely not active in both sensing and actuating processes. However, one could conceive an 'intelligent' bio-resorbable material having much more effective performances by using the concept developed in $[69,70,75,39,23,79]$. In literature, several strategies to obtain a parameterization of equilibrium shapes of bone-like structures can be found. Specifically, Cuomo et al. adopt the force density method to define the initial equilibrium configuration of cable-struts structures [42]. In this way, an optimization procedure based on the force density parameters can be performed analogously to [26]. In this context, it is also relevant the study performed in [16].

Some authors presented a model of growth and mass transfer in multi-constituent materials in which the variations of mass of the constituents, and the related structural reorganization of the material, were accompanied by inelastic deformations and determined the rates of these on the basis of thermodynamic arguments, see e.g. [43,46,45,44].

The remodeling process in bones and bio-materials due to the bio-mechanical evolution is similar to phase transitions and thus could be treated with the same theoretical tools $[65,27,28]$. Furthermore, the remodeling process presents some stability and bifurcation problems, especially when the applied load reaches a certain level in the presence of geometric or material nonlinearities. In a few papers, for cases of similar complexity, these difficulties are treated $[51,52,78,53,54]$. Only a limited number of analytical and experimental publications on viscoelastic behavior of living bone, may be found in the literature. However, in the authors' opinion, dissipation should be taken into account and, for this purpose, the analysis presented in $[14,13]$ is to be considered. Moreover, bones present micro-cracks or other damage mechanisms that can be described by means of second gradient continuum damage models (see e.g. [66, $58])$.

Lekszycki and dell'Isola in [49] proposed a model where a solid mixture is presented to describe phenomena of synthesis and of resorption for bioresorbable materials grafted in bone. The phenomena have been analyzed introducing evolution rules for mass densities describing the process of solid mixture forming/resorption activated by mechanical stimuli. In particular, Madeo et al. in [57] introduced a twoconstituent porous continuum as a model describing the growth/resorption processes in bone tissues grafted with bioresorbable materials as driven by mechanical stimulus, under the assumption of firstorder deformation theory. Madeo et al. in [56] presented an improvement of the previous model [57] under the assumption of second gradient deformation theory. Some numerical simulations for rod-like bones subjected to axial external load are presented in [57,56]. Lekszycki and dell'Isola in [49] proposed a continuum poro-elastic mixture model which applies to 3D case and performed numerical investigations in unidimensional samples to study mechanisms observable during remodeling processes. Andreaus et al. [7] employed the above 3D theoretical model, previously developed and used in 1D space [57,49], to investigate more complex geometries: a continuum model of a mixture of bone tissue and bio-resorbable material was in fact applied to 2D samples. Andreaus et al. [8] added the possibility of explicitly accounting for the presence of porosity in the bone/biomaterial mixture and for the presence of what we call a 'lazy zone'; the change of volume fraction related to the void volume is directly accounted for by considering porosity as an independent kinematical field. In recent years, synthetic grafts have been developed as substitutions of bone grafts. Thus, tissue engineering research in bone has focused on artificial and structural bone replacement and bone graft. On the basis of many of the papers mentioned above, which stressed the importance of the influence of different loading conditions on the phenomena of evolution of bone tissue under mechanical stimulus, this article aims to analyze and compare the effects of shear and pure bending on the remodeling process in 2D sample constituted by two elements, one of which is bone tissue and the other an artificial bio-resorbable material. In the framework of the Mixture theory, we consider a biphasic mixture deformable porous medium whose mass may vary in time (e.g., see [71, $67,55])$. Since this composite medium can be intended as a solid material with small, distributed voids, the linear theory of elastic materials with voids developed by Cowin and Nunziato [19] is employed to 
describe the mechanical behavior of such a mixture, where the change in void volume -introduced as an additional degree of kinematic freedom- is induced by deformations.

\section{Modeling}

The system in study is a mixture constituted by two elements, one of which is bone tissue and the other an artificial material of the kind used in bone reconstructive surgery. Initially these two constituents are located at distinct zones. It occurs that such an artificial material can be resorbed by actor cells, i.e. osteoclasts, which arrive in pores of the artificial material far away from bone-material interface. Furthermore, on the implant side the presence of bone tissue can be also observed after a while because of the activity of cells that synthesize bone, i.e. osteoblasts. There could be, thus, on this side a total or partial substitution of artificial material with bone. The sensor cells inside bone tissue are highly mechano-sensitive and drive the actor cells, which are responsible for the remodeling of the mixture, producing a stimulus that is assumed to depend mainly on the strain energy density.

The mechanical behavior of porous solids, such as bones or artificial grafts of bioresorbable material, cannot be described by means of the classical theory of elasticity. Following the approach of Mindlin [60] and Eringen [29] which introduced extended kinematics to study materials with microstructure, we consider a continuum model with micro-structure aiming at describing some of the porosity effects occurring in bone-like media [8]. Reconstructed bone is a material with microstructures which present high-contrast in microscopic properties; thus, to produce generalized continuum model, standard homogenisation techniques need to be modified and generalized in order to be adapted to the process of identifying the macro-properties of this material [24,25,62,61].

In accordance with the model developed by Cowing and Nunziato [19] for porous solids with voids and in addition to the displacement field $u_{i}(\boldsymbol{x}, t)$, we introduce a new independent kinematic degree of freedom: the change in volume fraction from the reference volume fraction, namely

$$
\xi(\boldsymbol{x}, t)=\left(\varsigma_{\mathrm{b}}+\varsigma_{\mathrm{m}}\right)-\left(\varsigma_{\mathrm{b}}^{*}+\varsigma_{\mathrm{m}}^{*}\right)
$$

where $\varsigma_{\mathrm{b}}=\rho_{\mathrm{b}} / \rho_{\mathrm{Max}}$ and $\varsigma_{\mathrm{m}}=\rho_{\mathrm{m}} / \rho_{\mathrm{Max}}$ are the volume distribution functions or volume fractions of bone and bioresorbable material, respectively; $\rho_{\mathrm{b}}$ and $\rho_{\mathrm{m}}$ being the apparent mass densities of the phases of which the mixture is composed and $\rho_{\mathrm{Max}}$ is the mass density of the mineral matrix, i.e. the maximal density without pores, assumed equal for the two phases. The superscript ' $*$ ' represents the quantity in the reference configuration. It is worth observing that the matrix density, $\rho_{\mathrm{Max}}$, in this work is not considered as a constant passing from the reference to the actual configuration, and consequently we do not introduce the constraint of incompressible distributed volume. According to the conventional notation, the porosity can be expressed as follows:

$$
\varphi(\boldsymbol{x}, t)=1-\left(\varsigma_{\mathrm{b}}+\varsigma_{\mathrm{m}}\right)
$$

and, thus, the change in volume fraction from the reference configuration, $\xi$, is related to the porosity, $\varphi$, by

$$
\xi(\boldsymbol{x}, t)=\varphi^{*}-\varphi
$$

Assuming the material to be isotropic, and following standard arguments for material symmetry characterization [18], the stored energy density $\mathscr{E}$ associated with strain and void volume distortion from the reference configuration can be chosen as the following quadratic form: $[19,8]$

$$
\mathscr{E}=\frac{1}{2} \frac{Y\left(\rho_{\mathrm{b}}^{*}, \rho_{\mathrm{m}}^{*}\right) \nu}{(1-2 \nu)(1+\nu)}\left(E_{i i}\right)^{2}+\frac{1}{2} \frac{Y\left(\rho_{\mathrm{b}}^{*}, \rho_{\mathrm{m}}^{*}\right)}{(1+\nu)} E_{i j} E_{i j}+\frac{1}{2} K_{1}\left(\rho_{\mathrm{b}}^{*}, \rho_{\mathrm{m}}^{*}\right) \xi^{2}+\frac{1}{2} K_{2} \xi_{, i} \xi_{, i}+K_{3}\left(\rho_{\mathrm{b}}^{*}, \rho_{\mathrm{m}}^{*}\right) \xi E_{i i}
$$


The strain tensor $E_{i j}(\boldsymbol{x}, t)$ is the Green-Saint Venant strain tensor determined from the displacement field according to the classical method

$$
E_{i j}=\frac{1}{2}\left(u_{i, j}+u_{j, i}+u_{i, k} u_{k, j}\right)
$$

$Y$ and $\nu$ are Young's modulus and Poisson's ratio, respectively; moreover $K_{1}, K_{2}$ and $K_{3}$ are the material parameters that take into account the presence of the voids. The comma followed by an index indicates a partial derivative with respect to the spatial coordinate. Herein, the elastic properties of the porous solid are given and the effect of the pores is lumped into the corresponding material constants. Alternatively, these properties could be estimated analytically from the elastic properties of the solid matrix, the geometry of the pores and the porosity, see, e.g. [33]. It has been noted that the most convenient pair of elastic constants for the expression of the stored energy density of an isotropic material is that comprised of the bulk modulus $\kappa$ and the shear modulus $\mu[76,77,35,37,32,34,36]$, even more so than the pair of Lamé's constants [36]. However, considering the example of application in Section 3, which employs a simple rectangular sample, we preferred to use the usual pair $Y-\nu$ of Strength of Materials. The isotropic material model was considered appropriate for a first study, since the use of more complex orthotropic material properties has been shown to have only a small influence on the Finite Element results [64]. Interface conditions can be imposed at the boundary between bone and material zones, $\partial \mathcal{B}_{\text {int }}$, by assuming an energy density

$$
\mathscr{E}_{\text {int }}=\frac{1}{2} K_{4}\left(\xi^{+}-\xi^{-}\right)^{2}=\frac{1}{2} K_{4} \llbracket \xi \rrbracket^{2} \geqslant 0
$$

where $\llbracket \xi \rrbracket$ denotes the jump of $\xi$ through the boundary $\partial \mathcal{B}_{\text {int }}$. Alternatively, the bone-material interface could be modeled with material properties, see e.g. $[22,21,68]$. In this case, if inertia effects are neglected, we can define the action functional $\mathcal{A}$ for the porous system as:

$$
\mathcal{A}:=-\int_{t_{0}}^{t_{1}} \int_{\mathcal{B}} \mathscr{E} \mathrm{d} \mathcal{V} \mathrm{d} t-\int_{t_{0}}^{t_{1}} \int_{\partial \mathcal{B}_{\mathrm{int}}} \mathscr{E}_{\mathrm{int}} \mathrm{d} \mathcal{S} \mathrm{d} t+\int_{t_{0}}^{t_{1}} \int_{\partial \mathcal{B}} \tau_{i} u_{i} \mathrm{~d} \mathcal{S} \mathrm{d} t+\int_{t_{0}}^{t_{1}} \int_{\partial \mathcal{B}} \Xi \xi \mathrm{d} \mathcal{S} \mathrm{d} t
$$

where the last two terms represent the work made on $u_{i}$ and $\xi$ by the actions externally applied at the boundary, $\tau_{i}$ and $\Xi$, respectively. By equating to zero the variation of the introduced Action, $\delta \mathcal{A}$, we obtain the mechanical governing equations of the system in terms of the kinematic degrees of freedom $u_{i}$ and $\xi$ :

$$
\begin{aligned}
\delta \mathcal{A}=-\int_{t_{0}}^{t_{1}} \int_{\mathcal{B}}\left[T_{i j} \delta E_{i j}+K_{1} \xi \delta \xi+K_{2} \xi_{, i} \delta \xi_{, i}+K_{3}\left(E_{i i} \delta \xi+\xi \delta E_{i i}\right)\right] \mathrm{d} \mathcal{V} \mathrm{d} t+ \\
-\int_{t_{0}}^{t_{1}} \int_{\partial \mathcal{B}_{\mathrm{int}}} K_{4} \llbracket \xi \rrbracket \delta \llbracket \xi \rrbracket \mathrm{d} \mathcal{S} \mathrm{d} t+\int_{t_{0}}^{t_{1}} \int_{\partial_{\tau} \mathcal{B}} \tau_{i} \delta u_{i} \mathrm{~d} \mathcal{S} \mathrm{d} t+\int_{t_{0}}^{t_{1}} \int_{\partial_{\Xi} \mathcal{B}} \Xi \delta \xi \mathrm{d} \mathcal{S} \mathrm{d} t=0
\end{aligned}
$$

where $\partial_{\tau} \mathcal{B}$ and $\partial_{\Xi} \mathcal{B}$ are the boundary on which the surface tractions $\tau_{i}$ and the generalized force $\Xi$ are respectively specified and being

$$
T_{i j}=2 \frac{Y\left(\rho_{\mathrm{b}}^{*}, \rho_{\mathrm{m}}^{*}\right)}{2(1+\nu)} E_{i j}+\frac{Y\left(\rho_{\mathrm{b}}^{*}, \rho_{\mathrm{m}}^{*}\right) \nu}{(1-2 \nu)(1+\nu)} E_{k k} \delta_{i j}
$$

Possibly, to make our formulation also applicable to the cases of non-integrable boundary forces, we can invoke the so-called Hamilton's Extended Principle (see, e.g., [59,9]). 


\subsection{Material parameters}

Material properties of the mixture are assumed inhomogeneous. Young's modulus $Y$ of the mixture varies with reference mass densities obeying the power-law model:

$$
Y=Y_{\mathrm{b} \operatorname{Max}}\left(\varsigma_{\mathrm{b}}^{*}\right)^{\beta_{\mathrm{b}}}+Y_{\mathrm{m} \operatorname{Max}}\left(\varsigma_{\mathrm{m}}^{*}\right)^{\beta_{\mathrm{m}}}
$$

where the maximal elastic moduli $Y_{\mathrm{b} \text { Max }}, Y_{\mathrm{m} \text { Max }}$ and exponents $\beta_{\mathrm{b}}, \beta_{\mathrm{m}}$ are constants. As a first approximation, we assume that the Poisson's ratio is constant $(\nu=0.3)$. In a similar way the material parameter $K_{1}$ is assumed such that

$$
K_{1}=K_{1 \mathrm{bMax}}\left(\varsigma_{\mathrm{b}}^{*}\right)^{\alpha_{\mathrm{b}}}+K_{1 \mathrm{mMax}}\left(\varsigma_{\mathrm{m}}{ }^{*}\right)^{\alpha_{\mathrm{m}}}
$$

where the maximal stiffness parameters $K_{1 \mathrm{~b} \text { Max }}, K_{1 \mathrm{~m} \text { Max }}$ and exponents $\alpha_{\mathrm{b}}, \alpha_{\mathrm{m}}$ are constants. In fact $K_{1}$ can be interpreted as a pore stiffness; while $K_{2}$ as a first approximation is assumed to be constant. On the other hand, material parameter $K_{3}$, whose physical interpretation is the coupling between strains and changes of volume fraction with respect to reference configuration, is assumed to depend on the reference porosity, $\varphi^{*}$, in order to attain the limit behavior of a classic elastic material when porosity vanishes, and monotonically to increase its effect when the porosity tends to one. It can be easily demonstrated that an equivalent Lamé parameter $\lambda_{\text {eq }}=\lambda-\lambda_{\mathrm{v}}$ can be introduced to consider the presence of voids [8]. Hence, we hypothesize that $\lambda_{\mathrm{v}}$ is a fraction of $\lambda$ depending on the reference porosity and thus $K_{3}$ is expressed by

$$
\lambda_{\mathrm{v}}=K_{3}^{2} / K_{1}=\hat{g}\left(\varphi^{*}\right) \lambda \quad \Rightarrow \quad \lambda_{\text {eq }}=\left(1-\hat{g}\left(\varphi^{*}\right)\right) \lambda \quad \Rightarrow \quad K_{3}=\sqrt{\hat{g}\left(\varphi^{*}\right) \lambda K_{1}}
$$

where the function $\hat{g}\left(\varphi^{*}\right)$ is non-negative and less than one. An example of function $\hat{g}\left(\varphi^{*}\right)$ can be assumed as follows:

$$
\hat{g}\left(\varphi^{*}\right)=A_{k_{3}}\left(\frac{1}{\pi} \operatorname{atan}\left[s_{k_{3}}\left(\varphi^{*}-\frac{1}{2}\right)\right]+\frac{1}{2}\right)-k_{03}
$$

where the parameter $A_{k_{3}}$ affects the amplitude of function $\hat{g}\left(\varphi^{*}\right)$ and ranges from 0 to 1 , the parameter $s_{k_{3}}$ has an effect on the slope of the curve and $k_{03}$ is a constant to nullify the value of the function $\hat{g}\left(\phi^{*}\right)$ when porosity vanishes.

\subsection{Evolution rules of growth and resorption}

It is well established that exercise results in increased bone mass, while unloading due to immobilization, bedrest, and weightlessness results in bone atrophy. This relationship between mechanical loading and bone remodeling was first postulated by Wolff in 1892. Several hypotheses exist explaining the mechanism involved in transmitting the signal from mechanical loading to the bone cells $[47,11,12]$. It is worth noting that bones are continuously remodeled by the coordinated action of bone-resorbing osteoclasts and boneforming osteoblasts. Herein the activity of osteoclasts and osteoblasts is triggered by a mechanical stimulus from sensor cells, the osteocytes. This stimulus involves signals, sent from osteocytes to the actor cells, that decrease exponentially with increasing distance; the actor cells elaborate and integrate all signals sent by surrounding sensor cells which reach them. In this study, the velocity of propagation of the stimulus signal through space is usually not considered; the velocity of signal is assumed to be infinite. Moreover, the sensor cells can only be located close to real living tissue (not in artificial graft) and the number of the actor cells in a given place depends on the porosity of host composite material, i.e. $\varphi$. These biological signals are able to trigger bone remodeling by directing osteoblastic activity and osteoclastic resorption. The peculiarity of the approach employed here but proposed by $[49,57,7]$, is to model all these signals with a stimulus function expressed in an integral form:

$$
S(\boldsymbol{x}, t)=\int_{\mathcal{B}} \mathscr{E}\left(\boldsymbol{x}_{0}, t\right) \varpi\left[\rho_{\mathrm{b}}\left(\boldsymbol{x}_{0}, t\right)\right] e^{-\frac{\left\|\boldsymbol{x}-\boldsymbol{x}_{0}\right\|^{2}}{2 D^{2}}} \mathrm{~d} \boldsymbol{x}_{0}-P_{\text {ref }}=P(\boldsymbol{x}, t)-P_{\text {ref }}
$$


where $D$ is the the range of action of osteocytes, $\varpi\left(\rho_{\mathrm{b}}\right)=\eta \rho_{\mathrm{b}} / \rho_{\mathrm{Max}}$ is the number of active sensor cells over the number of potentially available osteocytes, $P_{\text {ref }}$ is an activation threshold, associated with a biological equilibrium state for which the effect of resorption and synthesis are balanced and $\mathscr{E}$ represents the energy density of the solid mixture, which is directly affected by the porosity through the new variable introduced $\xi$ [8]. It is worth to mention that the law (14) makes the model non-local. (It should be noted that this concept was first conceived in Gabrio Piola's works and revealed by the recent paper [20]). Furthermore, the mixture is inhomogeneous and thus the material parameters, $Y, K_{1}$ and $K_{3}$, are variable and depend on the mass density of the bone and of the bio-resorbable material. Finally, we consider the evolution equations, that are coupled with the mechanical equations obtained from the variation of the functional defined by the energy (4), and describe the linkage between the rates of the two mass densities and local values of the stimulus function expressed in the integral form (14):

$$
\left\{\begin{array}{l}
\dot{\rho}_{\mathrm{b}}^{*}=A_{\mathrm{b}}(S) H(\varphi) \\
\dot{\rho}_{\mathrm{m}}^{*}=A_{\mathrm{m}}(S) H(\varphi)
\end{array}\right.
$$

where the functions $A_{\mathrm{b}}$ and $A_{\mathrm{m}}$ are piece-wise linear functions with different slopes for positive and negative values of stimulus (consider that $A_{\mathrm{m}}$ for positive values of the stimulus does not have any effect, as there cannot be synthesis of bio-material). In particular, the following forms for the functions $A_{\mathrm{b}}$ and $A_{\mathrm{m}}$ have been chosen:

$$
\begin{aligned}
& A_{\mathrm{b}}(S)= \begin{cases}s_{\mathrm{b}} S & \text { for } S \geqslant 0 \\
r_{\mathrm{b}} S & \text { for } S<0\end{cases} \\
& A_{\mathrm{m}}(S)= \begin{cases}0 & \text { for } S \geqslant 0 \\
r_{\mathrm{m}} S & \text { for } S<0\end{cases}
\end{aligned}
$$

The weight function $H$ is a parabola, i.e. $H=4 \varphi(1-\varphi)$. We remark that the current porosity, $\varphi$, is involved in the evolution Eqs. (15) because the mechanosensory cells of bone detect the state of porosity in the instantaneous configuration.

\section{Numerical simulations}

The analytical model presented above has been applied to a two-dimensional rectangular sample, (see Fig. 1). The domain $\mathcal{C}=[0, L] \times[0, W]$ denotes the region occupied by the mixtures. In detail, $L=2.5$ $\mathrm{mm}, W=1.00 \mathrm{~mm}$. Initially, the mass density distributions of the bone and of the graft material are taken constant and in such a way to consider the two constituents separated in order to deal with a simple but significant case for orthopedic and dental applications of bone graft. Let $\mathcal{C}_{b}=\left[0, L_{\mathrm{b}}\right] \times[0, W]$ and $\mathcal{C}_{m}=\left[L_{\mathrm{b}}, L_{\mathrm{b}}+L_{\mathrm{m}}\right] \times[0, W]$, with $L_{\mathrm{b}}=L_{\mathrm{m}}=L / 2$, be respectively the bone side and the graft side. Hence, initial conditions for $\rho_{\mathrm{b}}$ and $\rho_{\mathrm{m}}$ are

$$
\begin{cases}\rho_{\mathrm{b}}(\boldsymbol{X}, 0)=\rho_{\mathrm{b} 0}=\rho_{\mathrm{Max}} / 2 & \forall \boldsymbol{X} \in \mathcal{C}_{b} \\ \rho_{\mathrm{m}}(\boldsymbol{X}, 0)=\rho_{\mathrm{m} 0}=\rho_{\mathrm{Max}} / 2 & \forall \boldsymbol{X} \in \mathcal{C}_{m}\end{cases}
$$

The values of the constitutive parameters are reported in Tables 1 and 2 . In the figures of Sect. 3 the functions under exam are plotted along Cauchy cuts, $C_{\mathrm{bm} 1}(y=W / 4), C_{\mathrm{bm} 2}(y=3 W / 4)$ and in probe points $P_{\mathrm{m} 1}=(7 \mathrm{~L} / 8 ; \mathrm{W} / 4)$ and $P_{\mathrm{m} 2}=(7 \mathrm{~L} / 8 ; 3 \mathrm{~W} / 4)$. In all that follows, mass densities are normalized to the $\rho_{\text {Max }}=1800 \mathrm{~kg} / \mathrm{m}^{3}$, the stimulus is normalized with respect to $P_{\text {ref }}=1.98 \times 10^{-3} \mathrm{~J}$, and the Lamé parameters $\lambda$ and $\lambda_{\text {eq }}$ (see [8]) are normalized with respect to $Y_{\mathrm{bMax}}$. The displacement boundary conditions are, $u_{1}\left(0, X_{2}, t\right)=0$ and $u_{2}(0,0, t)=0$ to avoid rigid motions. Here, two load-cases are considered: 

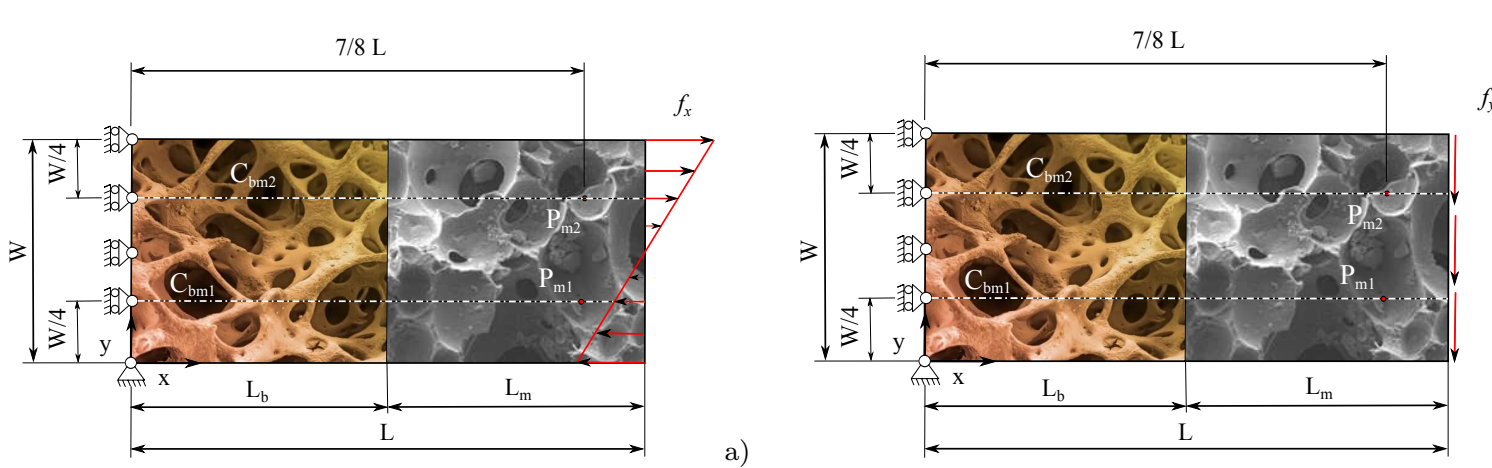

$f_{y}$

Fig. 1 Initial configuration, pure bending (a) and shear load (b).

Table 1 Values of the parameters used in numerical simulations.

\begin{tabular}{cccccccccc}
\hline$\rho_{\mathrm{b} \text { Max }}=\rho_{\mathrm{m} \text { Max }}$ & $Y_{\mathrm{b} \text { Max }}$ & $Y_{\mathrm{m} \mathrm{Max}}$ & $\beta_{\mathrm{b}}=\beta_{\mathrm{m}}$ & $\eta$ & $D$ & $\varepsilon_{\text {ref }}$ & $P_{\text {ref }}$ & $A_{k_{3}}$ & $s_{k_{3}}$ \\
\hline $1800 \mathrm{~kg} / \mathrm{m}^{3}$ & $14.0 \mathrm{GPa}$ & $11.2 \mathrm{GPa}$ & 2 & 0.2 & $0.1 L$ & $10^{-3}$ & $1.98 \times 10^{-3} \mathrm{~J}$ & 0.9 & 15 \\
\hline
\end{tabular}

Table 2 Values of the material coefficients used in numerical simulations.

\begin{tabular}{cccccccc}
\hline$K_{1 \mathrm{bMax}}$ & $K_{1 \mathrm{~m} \mathrm{Max}}$ & $\alpha_{\mathrm{b}}=\alpha_{\mathrm{m}}$ & $K_{2}$ & $K_{4}$ & $s_{\mathrm{b}}$ & $r_{\mathrm{b}}$ & $r_{\mathrm{m}}$ \\
\hline $140 \mathrm{GPa}$ & $112 \mathrm{GPa}$ & 2 & $8.75 \times 10^{3} \mathrm{~N}$ & $0.177 \mathrm{GPa}$ & $0.147 \mathrm{~s} / \mathrm{m}^{4}$ & $0.147 \mathrm{~s} / \mathrm{m}^{4}$ & $0.221 \mathrm{~s} / \mathrm{m}^{4}$ \\
\hline
\end{tabular}

- Bending case, in which the applied force has only one significant component along $x$ axis i.e. $f_{x}=$ $F_{x 0}(y / W-1 / 2)$ on the edge $x=L$;

- Shear case, in which the applied force has only one significant component along $y$ axis i.e. $f_{y}=F_{y 0}$ on the edge $x=L$ and it is constant.

According to [8], a 'lazy zone' was also accounted for, the width of which is defined as $w_{\mathrm{lz}}=(1+\delta) \varepsilon_{\text {ref }}$, where $\delta=5 \%$ and the reference homeostatic strain is $\varepsilon_{\text {ref }}=1000 \mu$ strain. In the figures of Sect. 3, the symbols $C$ and $T$ denote compression and tension respectively, and the arrows indicate the versus according to which time is incremented (Figs. 6 and 13).

The sequence of mechanical states of the considered bone-material sample were calculated via COMSOL Multiphysics ${ }^{\circledR}$, where a discrete time step increment algorithm is implemented. The mesh size was chosen as a suitable fraction $(1 / 6)$ of the influence distance $D$, to accomplish a numerical integration sufficiently accurate for the problem at hand. A 9-nodes quadrilateral finite element characterized by Lagrange interpolating polynomials of second order was chosen in order to optimize the discretization of the 2-D sample. The obtained results can be improved adopting the numerical formulation proposed in $[40,41,15]$ where the authors employ B-spline interpolations to formulate finite elements with high accuracy property, due to the high inter-element continuity and smoothness level insured by the blending of the interpolating polynomial functions. In addition, the formulation presented in [38,74] on a simple mixed-hybrid element for the linear analysis of Reissner-Mindlin plates, can be employed to avoid locking effects.

\subsubsection{Pure bending load condition}

In this subsection we study the case shown in Figure 1a where the load condition is represented by an affine axial force distribution with zero mean, applied at the right end $y=L$ and having maximum intensity $F_{x 0}=3.43 \times 10^{4} \mathrm{~N} / \mathrm{m}$. 


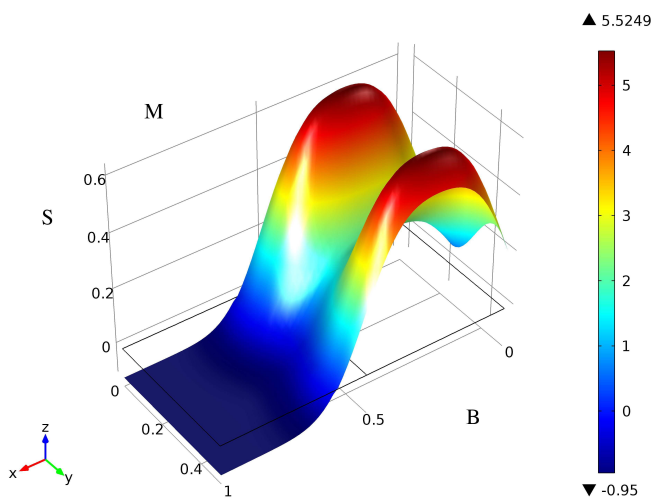

a)

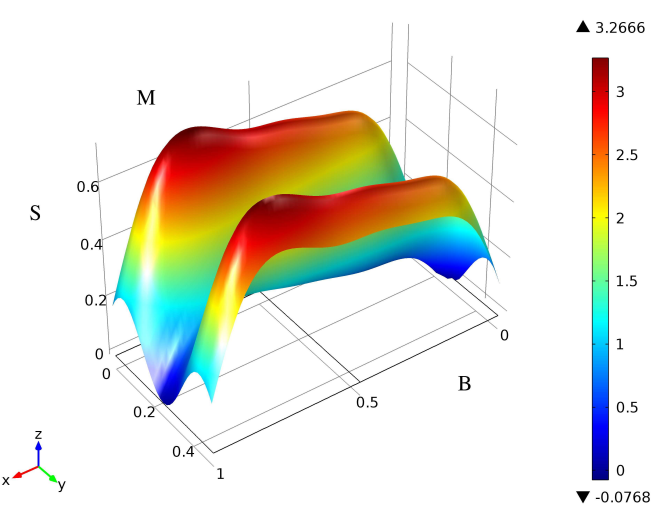

b)

Fig. 2 Distributions of Stimulus at the beginning (a) and at the end (b) of the process in bone (B) and material (M) zones.

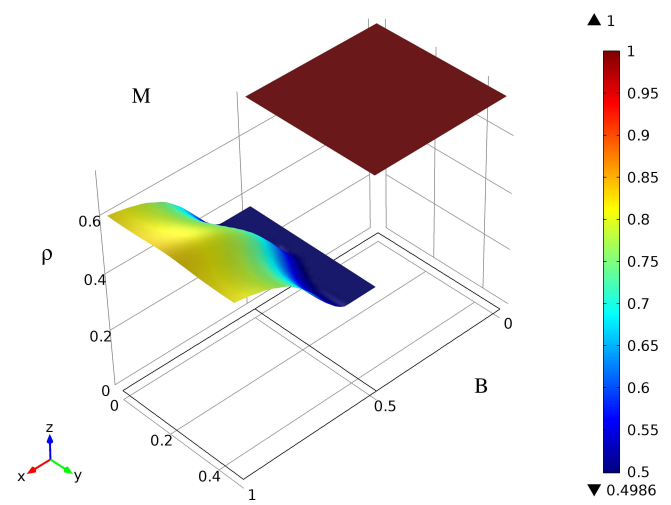

a)
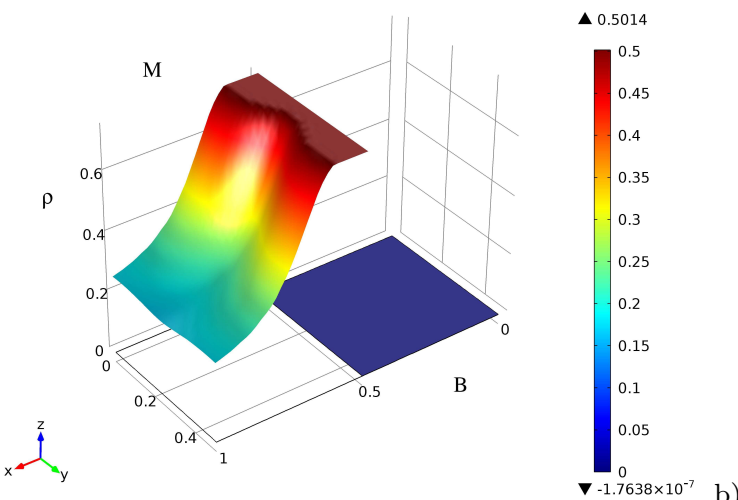

Fig. 3 Final distributions of mass densities of bone (a) and material (b) in bone (B) and material (M) zones.

Figure 2 shows the initial a) and final b) distributions of the stimulus, $S$, normalized to the threshold $P_{\text {ref, }}$, see Eq. (14). At the end of the process, these stimuli take only positive values and so the time rate of mass density is not negative. These values yield an equilibrium because the porosity is null and nothing can happen any longer since there is no more space available for an additional synthesis of bone. Figure 3 shows the final distributions of mass densities of bone a) and material b). It is observed in Fig. 2 that at the neutral axis (characterized by null axial stress) the stimulus is reduced, as indicated by the deep valley $(-15 \%)$ that can be seen at $y=W / 2$; therefore the material is reabsorbed; in the zone of the material (graft) a valley forms (see Fig. 3b), which is occupied by a ridge (-15\%) (see Fig. 3a) corresponding to the same zone. Since a Young's modulus $Y_{\mathrm{mMax}}<Y_{\mathrm{bMax}}$ has been assumed, it is observed that at the end of the process the stimulus in the area of material attained a level higher $(+38 \%)$ than that achieved in the area of the bone, where there is only bone, while in the area of the material the -more deformablemixture formed.

Figure 4 shows the initial and final distributions of the change in volume fraction $\xi$; it is worth noticing that the $\xi$ is positive in the compressed zone, whereas it is negative in tensioned zone. The effect of bending is visible throughout the domain, and at the end of the process the maximum value of $\xi$ is of an order of magnitude smaller with respect to the initial maximum value. It is noted that -at the end of the process - in the zone of the material (where the mixture formed) the $\xi$ is greater $(+66 \%)$ with respect to that one in the bone zone, and, that a greater deformation of the pores is experienced, because the 

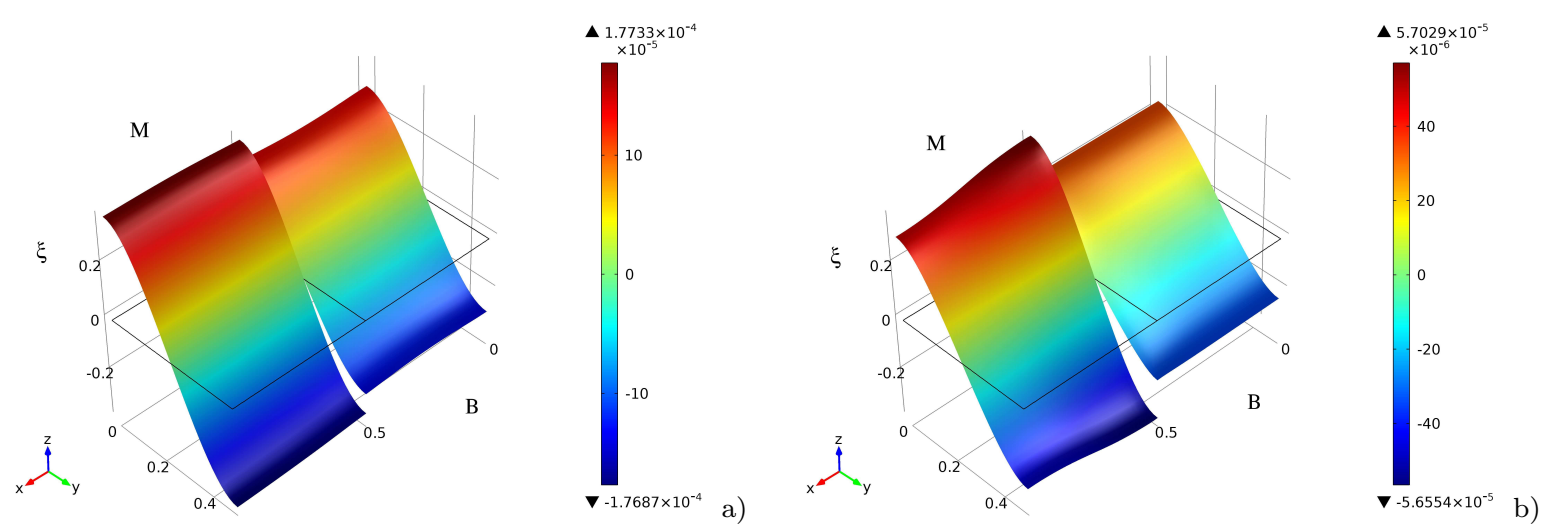

Fig. 4 Distributions of $\xi$ at the beginning (a) and at the end (b) of the process in bone (B) and material (M) zones.
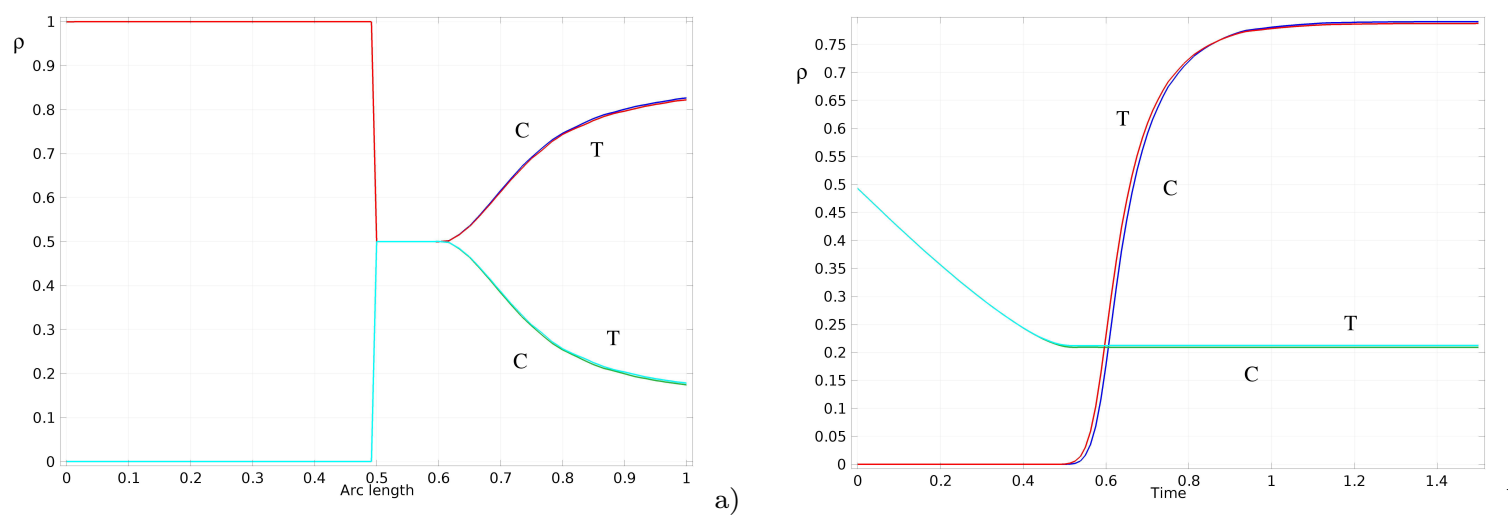

b)

Fig. 5 Distributions of mass densities of bone and material at the end of the process along Cauchy cuts (a) and time evolutions in probe points (b). ' $C$ ' and ' $T$ ' stand for 'Compression' and 'Tension', respectively.

material is less rigid than the bone. The discontinuity at the interface between the two zones is due to the elastic interaction between the two subdomains (see Eq. (6)).

Figure 5 shows distributions of mass densities of bone and material at the end of the process along Cauchy cuts, $C_{\mathrm{bm} 1}, C_{\mathrm{bm} 2}$ (a) and time evolutions in probe points $P_{\mathrm{m} 1}$ and $P_{\mathrm{m} 2}$ in the material zone (b). The two Cauchy cuts were made respectively in the compressed and in the tensioned zone. It can be seen in Fig. 5a that in the zone of the material the two upper curves are relative to the bone while the lower ones are relative to the material. The two curves relative to the two different Cauchy cuts (one in the compression zone and the other in the tension zone) are almost coincident. In Fig. 5b, it is observed a slight delay in the evolution time in the compression zone with respect to the tension one.

Figure 6 shows the time evolutions of the mass densities along the Cauchy cuts of the bone a) and of the material b). The arrows indicate the direction of the time increase. The thickening of the lines makes it possible to qualitatively estimate the speed at which the process takes place, knowing that curves are plotted at constant time step. In particular, it is observed that the phenomenon is fast at the beginning in the zone of the bone while, as regards the speed of bone growth, in the zone of the material we can distinguish four temporal phases: a rapid one at the beginning, followed by a slowing and then by an accelerating, concluding with a slow final saturation. In the zone of the material, a progression of the phenomenon of material resorption can be observed that occurs at almost constant speed. 

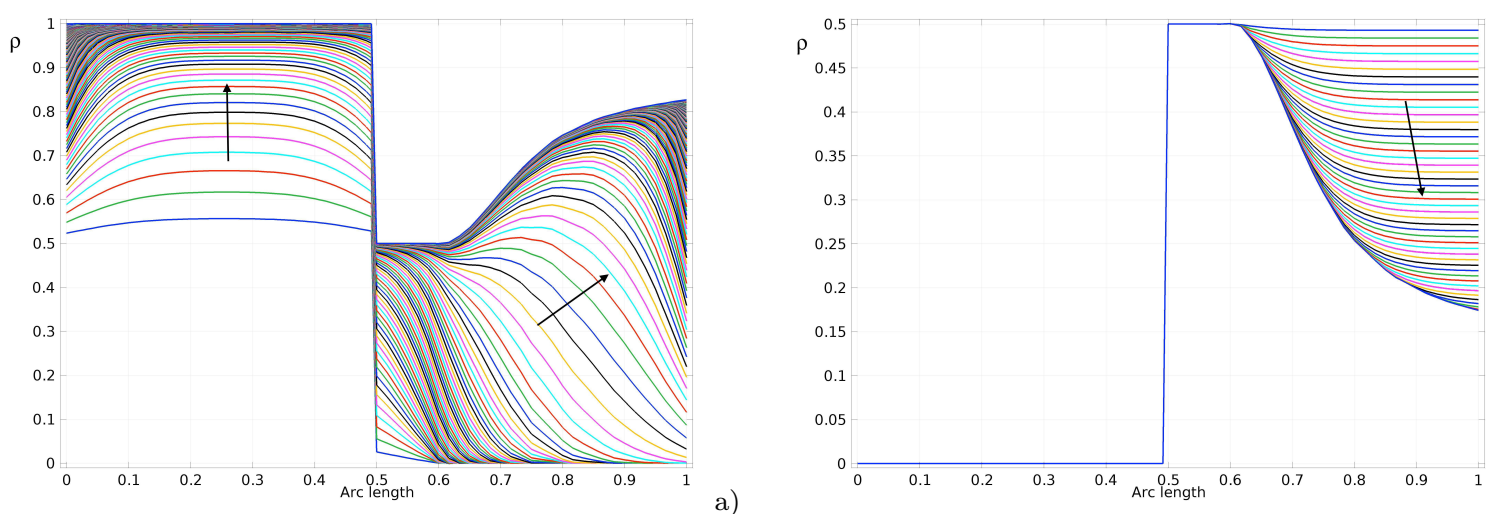

a)

Fig. 6 Time evolutions of mass densities along a Cauchy cut of bone (a) and material (b).
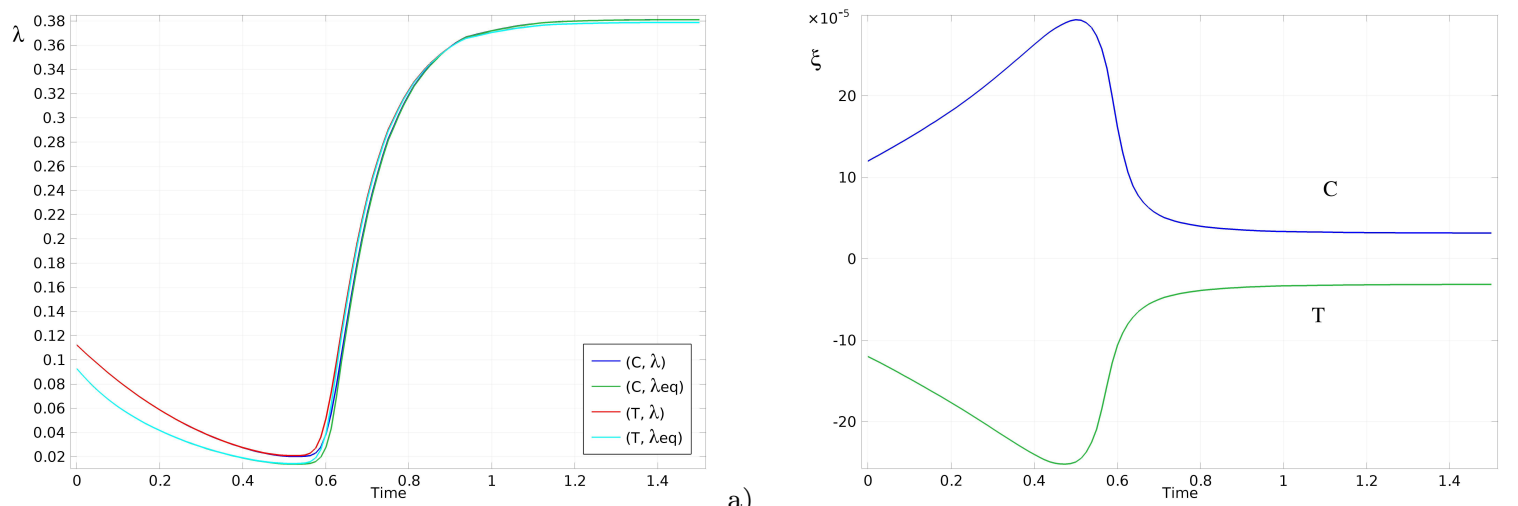

a)

Fig. 7 Time evolutions of $\lambda$ and $\lambda_{\text {eq }}(\mathbf{a})$ and time evolutions of $\xi(\mathbf{b})$ in $P_{m 1}$, i.e. the compressed zone, and $P_{m 2}$, i.e. the stretched zone. ' $C$ ' and ' $T$ ' stand for 'Compression' and 'Tension', respectively.

Figure 7a shows the times evolutions of $\lambda$ and $\lambda_{\text {eq }}$ in the probe points $P_{m 1}$ (compression side) and $P_{m 2}$ (tension side). It is observed that in $P_{m 1}$, i.e. the compressed zone, there is a slight delay, while at the beginning of the process $\left(\lambda_{\text {eq }}<\lambda\right)$ there is a more marked difference $(-35 \%)$ between the two parameters, which moreover realign at the end of the process. Figure $7 \mathrm{~b}$ shows the time evolutions of the $\xi$ in the two probe points $P_{\mathrm{m} 1}$ and $P_{\mathrm{m} 2}$; the upper curve at point $P_{\mathrm{m} 1}$ is in compression ( $\xi$ positive), while the lower curve ( $\xi$ negative) at point $P_{\mathrm{m} 2}$ is in tension. We note that the stationary values are slightly offset $(+11 \%)$ in time.

\subsubsection{Shear load condition}

In this subsection we study the case shown in Fig. 1b, where the load condition is represented by a uniform distribution of tangential force applied to the end $y=L$ and having intensity $F_{y 0}=3.43 \times 10^{3}$ $\mathrm{N} / \mathrm{m}$.

Figure 8 shows the initial a) and final b) distributions of the stimulus, $S$, normalized with respect

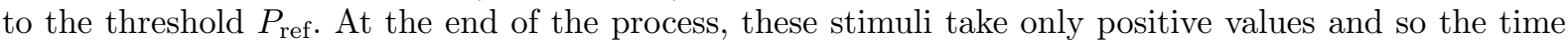
rate of mass densities is not negative. These values yield an equilibrium because the porosity is null and nothing can happen any longer. As already noted in respect of Fig. 2, also in this case we note again the influence of the neutral axis on the distribution of the stimulus, but now it is limited to the zone of the 


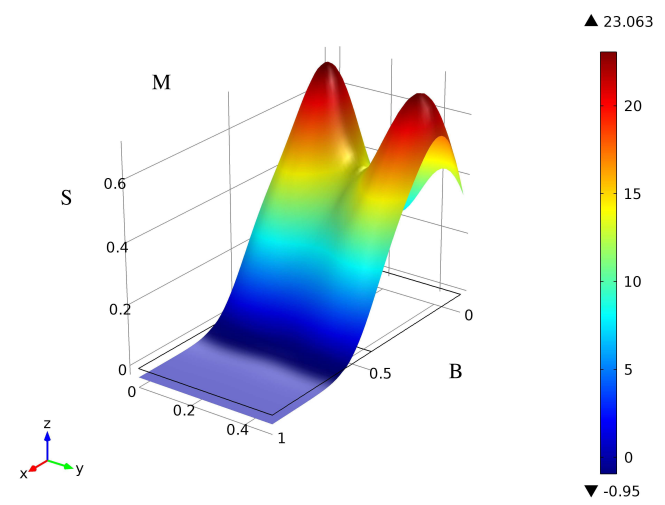

a)

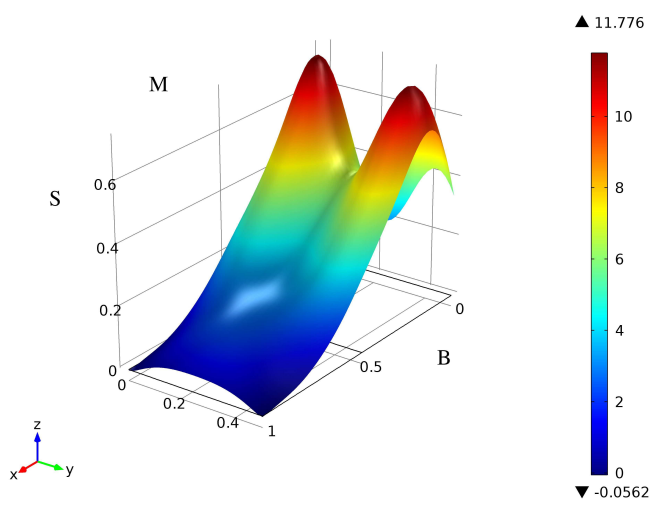

b)

Fig. 8 Distributions of Stimulus at the beginning (a) and at the end (b) of the process in bone (B) and material (M) zones.

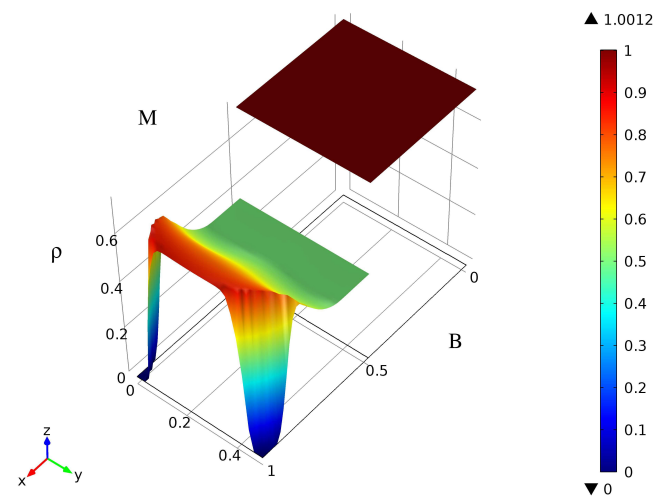

a)

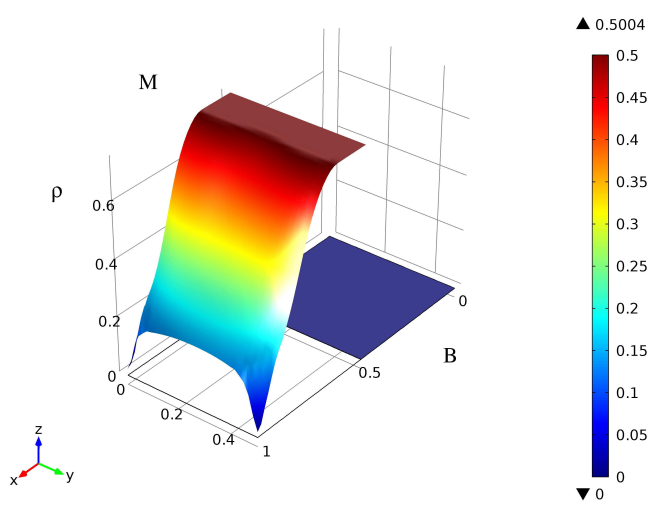

b)

Fig. 9 Final distributions of mass densities of bone (a) and material (b) in bone (B) and material (M) zones.

bone. Figure 9 shows the final distributions of the mass densities of the bone a) and of the material b) at the end of the process.

It may be noted, by comparing with the situation of Fig. 3, that the effect of the neutral axis (valley in the mass density of the material and the ridge in bone mass density) is much less evident than in the case of pure bending. It is observed that in the edges $x=L$ and $y=\{0, W\}$ the material is completely resorbed at the end of the process and the bone, in the absence of stimulus, as shown in Fig 8b, does not go to occupy the void, so that it remains unfilled. From a physiological point of view this is a situation to be avoided.

Figure 10 shows the distributions of the $\xi$ at the beginning a) and of the end b) of the process. It can be seen that close to the area of application of the load $\xi$ is almost constant and approximately null, because $\xi$ is governed by the axial stress and near the zone of application of the load the tangential stress is widely prevalent. In contrast, on the constrained side $x=0$, the effect of the bending moment is significant and therefore the effect of the linearity of the axial stress clearly does manifest itself. At the end of the process, the maximum value of $\xi$ is reduced to about $1 / 4$ of the maximum value of $\xi$ at the beginning of the process.

Figure 11 shows the distributions of the mass densities of the bone and of the material at the end of the process along Cauchy cuts $C_{\mathrm{bm} 1}$ (compression side) and $C_{\mathrm{bm} 2}$ (tension side) (a) and the times evolutions in probe points $P_{\mathrm{m} 1}$ (compression side) and $P_{\mathrm{m} 2}$ (tension side) (b). In Fig. 11 a more pronounced difference between compression and tension compared to the case of bending can be seen by making a comparison 


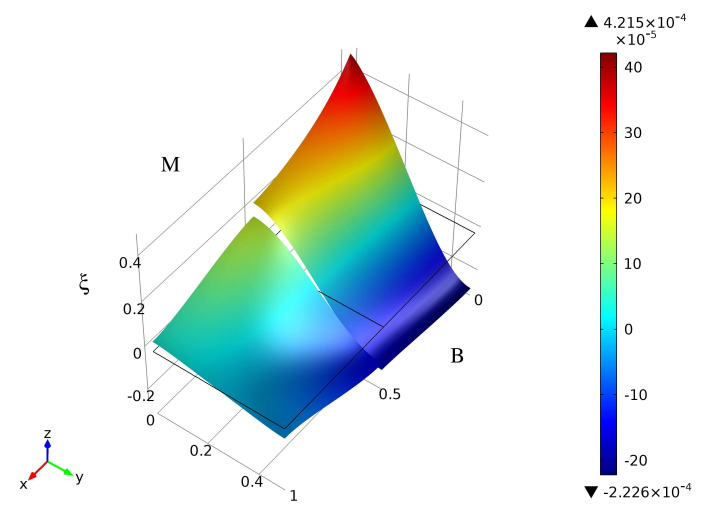

a)

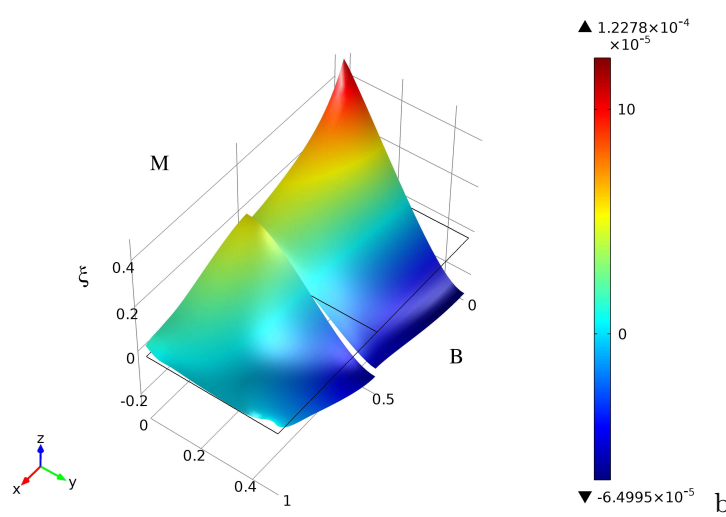

b)

Fig. 10 Distributions of $\xi$ at the beginning (a) and at the end (b) of the process in bone (B) and material (M) zones.
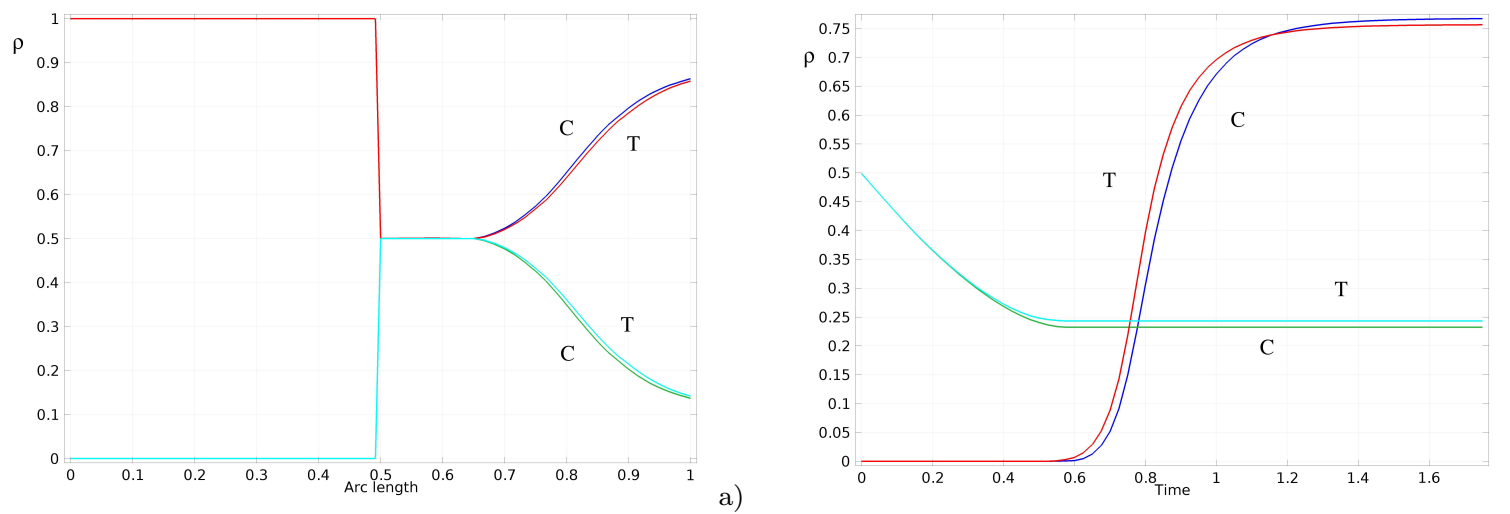

a)

Fig. 11 Distributions of mass densities of bone and material at the end along Cauchy cuts (a) and time evolutions in probe points (b). ' $C$ ' and ' $T$ ' stand for 'Compression' and 'Tension', respectively.

with Figure 5. Outer curves refer to the compression $\left(C_{\mathrm{bm} 1}\right)$ while the innermost curves refer to tension $\left(C_{\mathrm{bm} 2}\right)$. As can be seen from Fig. 11a the zone in which the material remains unabsorbed (near the interface) is more extensive (about two times) than in the case of the pure bending of Fig. 5a. From Fig. 11b it can be observed that the tension causes an advance in bone formation in the rapid (and steep) initial phase, while in the stationary situation leads to a slightly greater mass density.

Figure 12a shows the time evolutions of $\lambda$ and $\lambda_{\mathrm{eq}}$ in the probe points $P_{\mathrm{m} 1}$ e $P_{\mathrm{m} 2}$. We note a similar trend to that of the case of pure bending, comparing with Fig. 7, with the a marked differences between the two probe points; In the tension zone there is an advance in increasing the two parameters $\lambda$ and $\lambda_{\text {eq }}$ $\left(\lambda_{\text {eq }}<\lambda, \sim-45 \%\right)$ at the beginning of the process. Figure 12b shows the time evolutions of the $\xi$ in the two probe points $P_{\mathrm{m} 1}$ e $P_{\mathrm{m} 2}$. The upper curve is in the compression zone and the lower one in the tensile zone. It is noted that the difference between the abscissas of the points of stationarity is more marked $(+150 \%)$ than in the case of bending by comparing with Fig. $7 \mathrm{~b}$.

Figure 13 shows the time evolutions of the mass densities along the Cauchy cuts of the bone a) and of the material b). Observing Fig. 13a, we note that, in the zone of the bone, growth curves of bone are not uniform and that the maximum is shifted toward the constrained edge. This can be explained by observing that the stimulus in Fig. 8 has the maximum moved towards just the same constrained side. But in the case of pure bending (Figs. 2 and 6a) a more uniform growth is exhibited. Similar considerations 

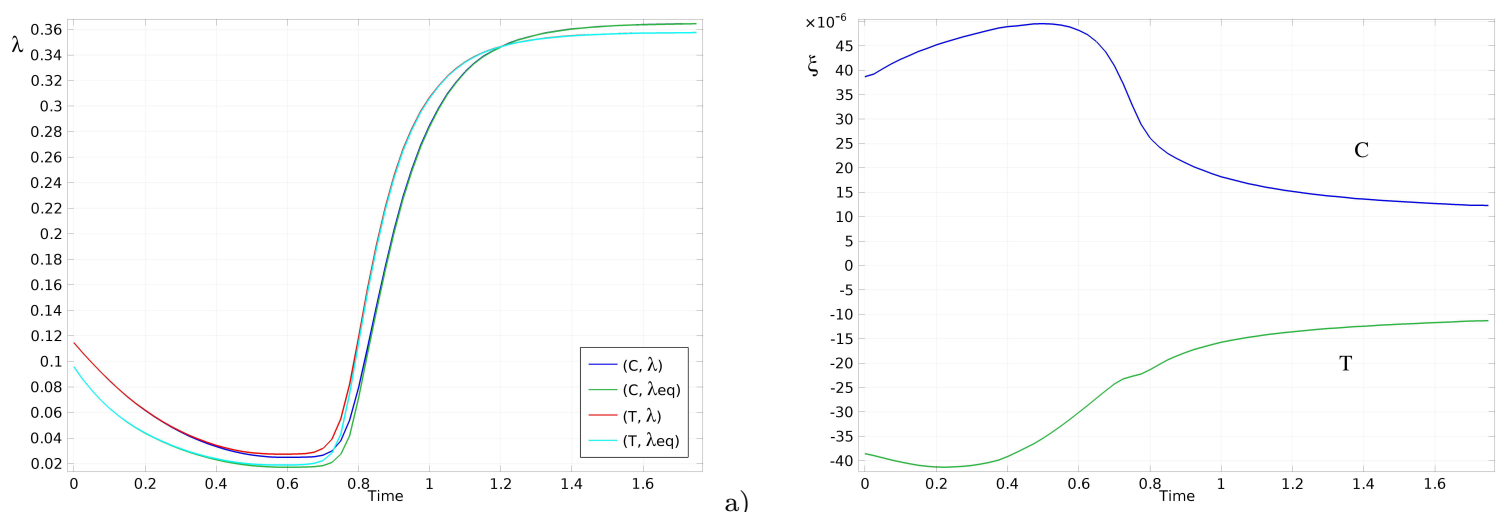

Fig. 12 Time evolutions of $\lambda$ and $\lambda_{\text {eq }}(\mathbf{a})$ and time evolutions of $\xi(\mathbf{b})$ in probe points. ' $C$ ' and ' $T$ ' stand for 'Compression' and 'Tension', respectively.
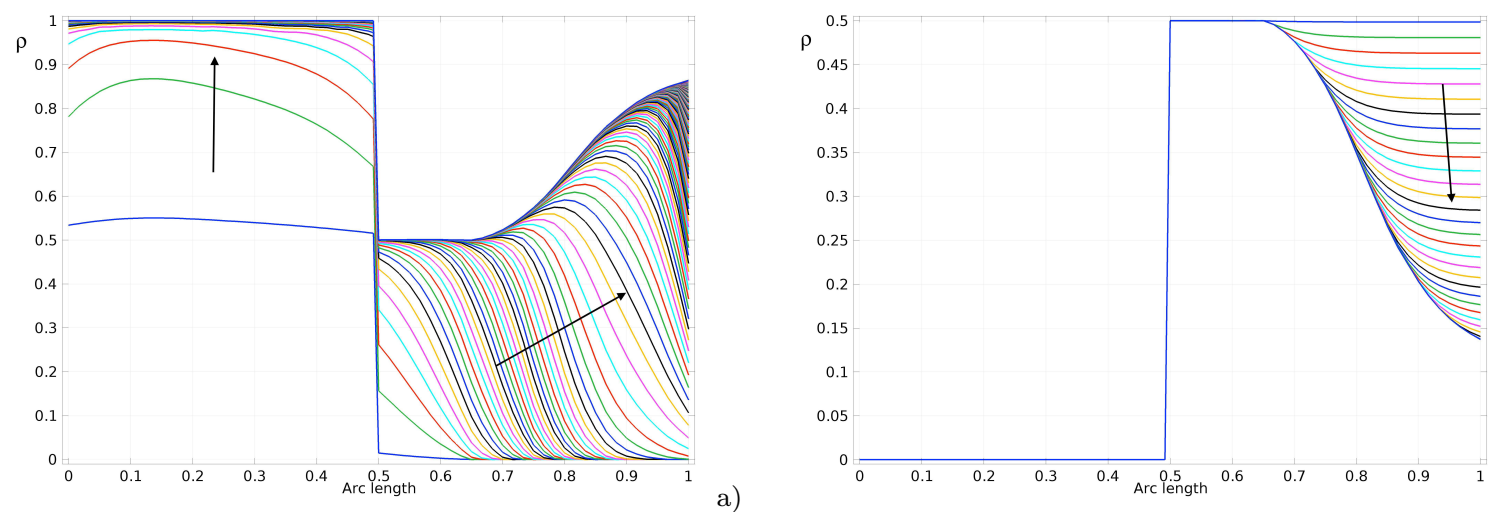

a)

Fig. 13 Time evolutions of mass densities of the bone (a) and the material (b) along a Cauchy cut.

to those made in respect of Figs. $6 \mathrm{a}$ and $6 \mathrm{~b}$ can be repeated also on the subject of Figs. $13 \mathrm{a}$ and $13 \mathrm{~b}$ as regards the rate of bone growth and resorption of the material.

\section{Discussion and conclusions}

In this paper, we use a mathematical model of a two-constituent mixture for describing bone tissue synthesis and resorption in presence of a bio-resorbable material as driven by mechanical loads. Porosity of bone tissue and bio-material mixture is accounted for, as it plays a relevant role in the biological activity of actor cells. In order to investigate and explain some of the possible mechanisms of interaction of bone tissue and bio-material, a simple two-dimensional case has been formulated and studied. At the initial time the mass density distributions of the bone and of the graft material are taken constant and in such a way the two constituents considered to be separated in order to deal with a simple but significant case for orthopedic and dental applications of bone graft. In detail, the left region is occupied by bone tissue and the right region by bio-resorbable material. As for boundary conditions, the sample is clamped on the left end. To see how and to what extent different external loads influence the process of interaction between bio-material and bone tissue, in some numerical examples, we considered a pure bending load and a shear load applied to the right end side. In particular, it is shown that, in the framework of this model, one can predict when rarefaction is prevented in bone tissue/bio-material composite and 
when the gradual substitution of the bio-material with bone living tissue is facilitated within appropriate ranges of bio-material properties. Due to consideration of the change of matrix volume fraction $\xi$ as an independent kinematic descriptor, which for this porous system takes the presence of voids into account, differences between compressive and tensile stresses result in different remodeling processes as well as in a significant difference of between the Lamé parameters $\lambda$ and $\lambda_{\text {eq }}$ in case of high porosity at the beginning of the process. The evolution process of bone growth in the bio-material zone is delayed in the case of compression with respect to the case of tension because of smaller porosity available for bone formation process due to an elastic contraction of pores. Observing Figs. 2 and 8, it is clear that inside the bio-resorbable material there is a partial substitution of graft material with bone. But comparing the two cases, it occurs that in shear case bio-material mass density is replaced by bone only in a narrow zone near the application of load, while in bending case this region is more extended (about 20\%). However, the level of substitution is higher $(+10 \%)$ in shear case with respect to the bending case.

The performed numerical simulations allowed for describing the most desirable situations in which a gradual resorption of the artificial material takes place together with the contemporary formation of new bone, finally giving rise to an almost complete replacement of the artificial material with natural living tissue.

The qualitative and quantitative results presented in this paper show that the enhanced model should be able to predict the occurrence of artificial material inclusions or voids in the completely healed reconstructed bones and possibly to optimize the material characteristics with respect to the best regeneration of bone, in relation to the different load conditions. Numerical simulations were worked out which provided ranges of the bio-mechanical parameters for which residual regions of bio-materials remain in remodeled bone and when almost all bio-material is completely replaced by bone tissue. In order to illustrate the potentialities of the model and of the method proposed in realistic clinical cases, some situations particularly interesting from the application point of view have been taken into consideration. These results could be useful to develop an optimal design of artificial grafts and to guide the choice and preparation of experimental tests.

Acknowledgements The authors would like to thank F. dell'Isola for helpful comments and advices through the study. A special thank to D. Steigmann for the interesting discussions in occasion of his stay in Rome during the summer 2013.

\section{References}

1. Andreaus, U., Colloca, M.: Prediction of micromotion initiation of an implanted femur under physiological loads and constraints using the finite element method. Proc Inst Mech Eng H 223(5), 589-605 (2009)

2. Andreaus, U., Colloca, M., Iacoviello, D.: An optimal control procedure for bone adaptation under mechanical stimulus. Control Engineering Practice 20(6), 575 - 583 (2012). DOI http://dx.doi.org/10.1016/j.conengprac.2012.02.002

3. Andreaus, U., Colloca, M., Iacoviello, D.: Modeling of Trabecular Architecture as Result of an Optimal Control Procedure, Lecture Notes in Computational Vision and Biomechanics, vol. 4. Springer Netherlands (2013)

4. Andreaus, U., Colloca, M., Iacoviello, D.: Optimal bone density distributions: numerical analysis of the osteocyte spatial influence in bone remodeling. Comput Methods Programs Biomed 113(1), 80-91 (2014). DOI 10.1016/j.cmpb.2013. 09.002

5. Andreaus, U., Colloca, M., Iacoviello, D., Pignataro, M.: Optimal-tuning pid control of adaptive materials for structural efficiency. Structural and Multidisciplinary Optimization 43(1), 43-59 (2011)

6. Andreaus, U., Colloca, M., Toscano, A.: Mechanical behaviour of a prosthesized human femur: a comparative analysis between walking and stair climbing by using the finite element method. Biophys. and Bioengin. Letters 1(3), 1-15 (2008)

7. Andreaus, U., Giorgio, I., Lekszycki, T.: A 2-D continuum model of a mixture of bone tissue and bio-resorbable material for simulating mass density redistribution under load slowly variable in time. ZAMM - Journal of Applied Mathematics and Mechanics / Zeitschrift für Angewandte Mathematik und Mechanik (2013). DOI 10.1002/zamm.201200182

8. Andreaus, U., Giorgio, I., Madeo, A.: Modeling of the interaction between bone tissue and resorbable biomaterial as linear elastic materials with voids. ZAMP - Zeitschrift für Angewandte Mathematik und Physik / Journal of Applied Mathematics and Physics pp. 1-29 (2014) 
9. Batra, G.: On hamilton's principle for thermo-elastic fluids and solids, and internal constraints in thermo-elasticity. Archive for Rational Mechanics and Analysis 99(1), 37-59 (1987)

10. Bevill, G., Eswaran, S.K., Farahmand, F., Keaveny, T.M.: The influence of boundary conditions and loading mode on high-resolution finite element-computed trabecular tissue properties. Bone 44(4), 573-8 (2009). DOI 10.1016/j.bone. 2008.11.015

11. Burger, E.H., Klein-Nulend, J.: Mechanotransduction in bone-role of the lacuno-canalicular network. The FASEB Journal 13(9001), S101-S112 (1999)

12. Burger, E.H., Klein-Nulend, J.: Responses of bone cells to biomechanical forces in vitro. Advances in dental research 13(1), 93-98 (1999)

13. Carcaterra, A., Akay, A.: Dissipation in a finite-size bath. Physical Review E - Statistical, Nonlinear, and Soft Matter Physics 84(1) (2011)

14. Carcaterra, A., Roveri, N., Pepe, G.: Fractional dissipation generated by hidden wave-fields. Mathematics and Mechanics of Solids (2014). DOI 10.1177/1081286513518941

15. Cazzani, A., Malagù, M., Turco, E.: Isogeometric analysis of plane-curved beams. Mathematics and Mechanics of Solids (2014). DOI 1081286514531265

16. Cazzani, A., Rovati, M.: Sensitivity analysis and optimum design of elastic-plastic structural systems. Meccanica 26(2-3), 173-178 (1991)

17. Chun, H., Shin, H., Han, C., Lee, S.: Influence of implant abutment type on stress distribution in bone under various loading conditions using finite element analysis. Int J Oral Maxillofac Implants 21(2), 195-202 (2006)

18. Cowin, S.C., Mehrabadi, M.M.: Anisotropic symmetries of linear elasticity. Applied Mechanics Reviews 48(5), 247-285 (1995)

19. Cowin, S.C., Nunziato, J.W.: Linear elastic materials with voids. Journal of Elasticity 13(2), 125-147 (1983)

20. dell'Isola, F., Andreaus, U., Placidi, L.: At the origins and in the vanguard of peridynamics, non-local and highergradient continuum mechanics: An underestimated and still topical contribution of Gabrio Piola. Mathematics and Mechanics of Solids (2014). DOI 10.1177/1081286513509811

21. dell'Isola, F., Kosinski, W.: Deduction of thermodynamic balance laws for bidimensional nonmaterial directed continua modelling interphase layers. Archives of Mechanics 45, 333-359 (1993)

22. dell'Isola, F., Romano, A.: On the derivation of thermomechanical balance equations for continuous systems with a nonmaterial interface. International Journal of Engineering Science 25, 1459-1468 (1987)

23. dell'Isola, F., Vidoli, S.: Continuum modelling of piezoelectromechanical truss beams: an application to vibration damping. Archive of Applied Mechanics 68(1), 1-19 (1998)

24. dell'Isola, F., Woźniak, C.: On continuum modelling the interphase layers in certain two-phase elastic solids. ZAMM Journal of Applied Mathematics and Mechanics / Zeitschrift für Angewandte Mathematik und Mechanik 77(7), 519-526 (1997)

25. dell'Isola, F., Woźniak, C.: On phase transition layers in certain micro-damaged two-phase solids. International Journal of Fracture 83(2), 175-189 (1997)

26. Descamps, B.: Computational Design of Lightweight Structures: Form Finding and Optimization. John Wiley \& Sons (2014)

27. Eremeyev, V.A., Pietraszkiewicz, W.: Phase transitions in thermoelastic and thermoviscoelastic shells. Archives of Mechanics 61(1), 41-67 (2009)

28. Eremeyev, V.A., Pietraszkiewicz, W.: Thermomechanics of shells undergoing phase transition. Journal of the Mechanics and Physics of Solids 59(7), 1395-1412 (2011). DOI http://dx.doi.org/10.1016/j.jmps.2011.04.005

29. Eringen, A.C.: Theory of thermo-microstretch elastic solids. International journal of engineering science 28(12), 1291$1301(1990)$

30. Farrar, D.: Bioresorbable polymers in orthopaedics. Medical Device Manufacturing \& Technology pp. 36-38 (2005)

31. Fatihhi, S.J., Ardiyansyah, S., Harun, M.N., Rabiatul, A.A.R., Jaafar, A., Afriwardi: Finite element simulation: The effects of loading modes at different anatomical sites of trabecular bone on morphological indices. Advanced Materials Research 845, 266-270 (2013)

32. Federico, S.: Volumetric-distortional decomposition of deformation and elasticity tensor. Mathematics and Mechanics of Solids 15(6), 672-690 (2009)

33. Federico, S.: On the linear elasticity of porous materials. International Journal of Mechanical Sciences 52(2), 175-182 (2010). DOI http://dx.doi.org/10.1016/j.ijmecsci.2009.09.006

34. Federico, S.: Covariant formulation of the tensor algebra of non-linear elasticity. International Journal of Non-Linear Mechanics 47(2), 273-284 (2012)

35. Federico, S., Grillo, A., Herzog, W.: A transversely isotropic composite with a statistical distribution of spheroidal inclusions: a geometrical approach to overall properties. Journal of the Mechanics and Physics of Solids 52(10), 2309$2327(2004)$

36. Federico, S., Grillo, A., Imatani, S.: The linear elasticity tensor of incompressible materials. Mathematics and Mechanics of Solids p. 1081286514550576 (2014)

37. Federico, S., Grillo, A., Wittum, G.: Considerations on incompressibility in linear elasticity. Il Nuovo cimento della Società italiana di fisica. C 32(1), 81 (2009)

38. Garusi, E., Tralli, A., Cazzani, A.: An unsymmetric stress formulation for reissner-mindlin plates: A simple and lockingfree rectangular element. International Journal of Computational Engineering Science 5(3), 589-618 (2004) 
39. Giorgio, I., Culla, A., Del Vescovo, D.: Multimode vibration control using several piezoelectric transducers shunted with a multiterminal network. Archive of Applied Mechanics 79(9), 859-879 (2009)

40. Greco, L., Cuomo, M.: B-Spline interpolation of Kirchhoff-Love space rods. Computer Methods in Applied Mechanics and Engineering 256(0), 251 - 269 (2013). DOI http://dx.doi.org/10.1016/j.cma.2012.11.017

41. Greco, L., Cuomo, M.: An implicit G1 multi patch B-spline interpolation for Kirchhoff-Love space rod. Computer Methods in Applied Mechanics and Engineering 269(0), 173-197 (2014). DOI http://dx.doi.org/10.1016/j.cma.2013. 09.018

42. Greco, L., Impollonia, N., Cuomo, M.: A procedure for the static analysis of cable structures following elastic catenary theory. International Journal of Solids and Structures 51(7), 1521-1533 (2014)

43. Grillo, A., Federico, S., Wittum, G.: Growth, mass transfer, and remodeling in fiber-reinforced, multi-constituent materials. International Journal of Non-Linear Mechanics 47(2), 388 -401 (2012). DOI http://dx.doi.org/10.1016/j. ijnonlinmec.2011.09.026

44. Grillo, A., Wittum, G.: Growth and mass transfer in multi-constituent biological materials. In: ICNAAM 2010: International Conference of Numerical Analysis and Applied Mathematics 2010, vol. 1281, pp. 355-358. AIP Publishing (2010)

45. Grillo, A., Wittum, G., Federico, S., Imatani, S., Giaquinta, G., Mićunović, M.V.: Evolution of a fibre-reinforced growing mixture. Nuovo Cimento C 32C(1), 97-119 (2009). DOI 10.1393/ncc/i2009-10356-1

46. Grillo, A., Wittum, G., Tomic, A., Federico, S.: Remodelling in statistically oriented fibre-reinforced materials and biological tissues. Mathematics and Mechanics of Solids (2014). DOI 1081286513515265

47. Klein-Nulend, J., Bacabac, R.G., Mullender, M.G.: Mechanobiology of bone tissue. Pathologie biologie 53(10), 576-580 (2005)

48. Klein-Nulend, J., Bakkerl, A.D., Bacabac, R.G., Vatsa, A., Weinbaum, S.: Mechanosensation and transduction in osteocytes. Bone 54, 182-190 (2013)

49. Lekszycki, T., dell'Isola, F.: A mixture model with evolving mass densities for describing synthesis and resorption phenomena in bones reconstructed with bio-resorbable materials. ZAMM - Journal of Applied Mathematics and Mechanics / Zeitschrift für Angewandte Mathematik und Mechanik 92(6), 426-444 (2012)

50. Li, L.H., Kommareddy, K.P., Pilz, C., Zhou, C.R., Fratzl, P., Manjubala, I.: In vitro bioactivity of bioresorbable porous polymeric scaffolds incorporating hydroxyapatite microspheres. Acta Biomater 6(7), 2525-31 (2010). DOI 10.1016/j.actbio.2009.03.028

51. Luongo, A.: Perturbation methods for nonlinear autonomous discrete-time dynamical systems. Nonlinear Dynamics 10(4), 317-331 (1996)

52. Luongo, A., Paolone, A.: Perturbation methods for bifurcation analysis from multiple nonresonant complex eigenvalues. Nonlinear Dynamics 14(3), 193-210 (1997)

53. Luongo, A., Piccardo, G.: A continuous approach to the aeroelastic stability of suspended cables in 1 : 22 internal resonance. JVC/Journal of Vibration and Control 14(1-2), 135-157 (2008)

54. Luongo, A., Zulli, D., Piccardo, G.: A linear curved-beam model for the analysis of galloping in suspended cables. Journal of Mechanics of Materials and Structures 2(4), 675-694 (2007)

55. Madeo, A., Djeran-Maigre, I., Rosi, G., Silvani, C.: The effect of fluid streams in porous media on acoustic compression wave propagation, transmission, and reflection. Continuum Mechanics and Thermodynamics 25(2-4), 173-196 (2013)

56. Madeo, A., George, D., Lekszycki, T.: A second gradient continuum model accounting for some effects of micro-structure on reconstructed bone remodelling. Comptes Rendus Mécanique 340(8), 575-589 (2012)

57. Madeo, A., Lekszycki, T., dell'Isola, F.: A continuum model for the bio-mechanical interactions between living tissue and bio-resorbable graft after bone reconstructive surgery. Comptes Rendus Mécanique 339(10), 625-640 (2011)

58. Madeo, A., Placidi, L., Rosi, G.: Towards the design of metamaterials with enhanced damage sensitivity: Second gradient porous materials. Research in Nondestructive Evaluation 25(2), 99-124 (2014)

59. Maugin, G.A.: Material inhomogeneities in elasticity, vol. 3. CRC Press (1993)

60. Mindlin, R.D.: Micro-structure in linear elasticity. Archive for Rational Mechanics and Analysis 16(1), 51-78 (1964)

61. Misra, A., Singh, V.: Micromechanical model for viscoelastic-materials undergoing damage. Continuum Mechanics and Thermodynamics 25, 1-16 (2013)

62. Misra, A., Yang, Y.: Micromechanical model for cohesive materials based upon pseudo-granular structure. International Journal of Solids and Structures 47, 2970-2981 (2010)

63. Olsson, T., Klarbring, A.: Residual stresses in soft tissue as a consequence of growth and remodeling: application to an arterial geometry. European Journal of Mechanics-A/Solids 27(6), 959-974 (2008)

64. Peng, L., Bai, J., Zeng, X., Zhou, Y.: Comparison of isotropic and orthotropic material property assignments on femoral finite element models under two loading conditions. Medical Engineering \& Physics 28(3), 227-233 (2006)

65. Pietraszkiewicz, W., Eremeyev, V., Konopińska, V.: Extended non-linear relations of elastic shells undergoing phase transitions. ZAMM - Journal of Applied Mathematics and Mechanics / Zeitschrift für Angewandte Mathematik und Mechanik 87(2), 150-159 (2007). DOI 10.1002/zamm.200610309

66. Placidi, L.: A variational approach for a nonlinear 1-dimensional second gradient continuum damage model. Continuum Mechanics and Thermodynamics pp. 1-16 (2014)

67. Placidi, L., dell'Isola, F., Ianiro, N., Sciarra, G.: Variational formulation of pre-stressed solid-fluid mixture theory, with an application to wave phenomena. European Journal of Mechanics - A/Solids 27(4), 582-606 (2008) 
68. Placidi, L., Rosi, G., Giorgio, I., Madeo, A.: Reflection and transmission of plane waves at surfaces carrying material properties and embedded in second-gradient materials. Mathematics and Mechanics of Solids 19(5), 555-578 (2014)

69. Porfiri, M., dell'Isola, F., Frattale Mascioli, F.M.: Circuit analog of a beam and its application to multimodal vibration damping, using piezoelectric transducers. International Journal of Circuit Theory and Applications 32(4), 167-198 (2004). DOI 10.1002/cta.273

70. Porfiri, M., dell'Isola, F., Santini, E.: Modeling and design of passive electric networks interconnecting piezoelectric transducers for distributed vibration control. International Journal of Applied Electromagnetics and Mechanics 21(2), 69-87 (2005)

71. Quiligotti, S., Maugin, G.A., dell'Isola, F.: An Eshelbian approach to the nonlinear mechanics of constrained solid-fluid mixtures. Acta Mechanica 160(1-2), 45-60 (2003)

72. Shi, X., Wang, X., Niebur, G.L.: Effects of loading orientation on the morphology of the predicted yielded regions in trabecular bone. Ann Biomed Eng 37(2), 354-62 (2009). DOI 10.1007/s10439-008-9619-4

73. Soltész, U.: The influence of loading conditions on the life-times in fatigue testing of bone cements. Journal of Materials Science: Materials in Medicine 5(9-10), 654-656 (1994)

74. Turco, E., Caracciolo, P.: Elasto-plastic analysis of kirchhoff plates by high simplicity finite elements. Computer methods in applied mechanics and engineering 190(5), 691-706 (2000)

75. Vidoli, S., dell'Isola, F.: Vibration control in plates by uniformly distributed PZT actuators interconnected via electric networks. European Journal of Mechanics - A/Solids 20(3), 435 - 456 (2001). DOI http://dx.doi.org/10.1016/ S0997-7538(01)01144-5

76. Walpole, L.J.: Elastic behavior of composite materials: theoretical foundations. Adv. Appl. Mech 21, 169-242 (1981)

77. Walpole, L.J.: Fourth-rank tensors of the thirty-two crystal classes: multiplication tables. Proceedings of the Royal Society of London. A. Mathematical and Physical Sciences 391(1800), 149-179 (1984)

78. Yeremeyev, V., Freidin, A., Sharipova, L.: The stability of the equilibrium of two-phase elastic solids. Journal of Applied Mathematics and Mechanics 71(1), 61 - 84 (2007). DOI http://dx.doi.org/10.1016/j.jappmathmech.2007.03.007

79. Zhu, X., Hao, H., Fan, K., Wang, Y., Ou, J.: Debond detection in RC structures using piezoelectric materials. pp. 261-262 (2009) 